Archives de sciences sociales des religions

112 | octobre-décembre 2000

Âme et corps : conceptions de la personne

\title{
Âme et corps dans l'Occident médiéval : une dualité dynamique, entre pluralité et dualisme
}

Jérôme Baschet

\section{OpenEdition}

Édition électronique

URL : http://journals.openedition.org/assr/20243

DOI : $10.4000 /$ assr.20243

ISSN : $1777-5825$

Éditeur

Éditions de l'EHESS

\section{Édition imprimée}

Date de publication : 31 décembre 2000

Pagination : $5-30$

ISBN : 2-222-96698-

ISSN : 0335-5985

\section{Référence électronique}

Jérôme Baschet, "Âme et corps dans l'Occident médiéval : une dualité dynamique, entre pluralité et dualisme ", Archives de sciences sociales des religions [En ligne], 112 | octobre-décembre 2000, mis en ligne le 19 août 2009, consulté le 01 mai 2019. URL : http://journals.openedition.org/assr/20243 DOI : 10.4000/assr.20243

Ce document a été généré automatiquement le 1 mai 2019.

() Archives de sciences sociales des religions 


\title{
Âme et corps dans l'Occident médiéval : une dualité dynamique, entre pluralité et dualisme
}

\author{
Jérôme Baschet
}

\section{NOTE DE L'ÉDITEUR}

Ce travail a été présenté lors du colloque franco-mexicain « Mundos de aquí, Mundos de allá. Confrontation des mondes. Histoire/Anthropologie/Sociologie comparées des sociétés européennes et mésoaméricaines ", organisé à San Cristóbal de las Casas (Chiapas, Mexique) du $1^{\mathrm{er}}$ au 4 juillet 1998. Conformément à la structure comparative de la rencontre, le thème « Conceptions de la personne (âmes et corps) » associait le présent travail, l'analyse anthropologique des conceptions tzeltales proposée par Pedro Pitarch Ramón, et le commentaire de Mario H. Ruz. Il importe cependant de souligner que la jonction des deux premiers textes, rassemblés dans la présente publication, ne prétend nullement constituer un véritable travail comparatif; il ne s'agit pas davantage de s'engager dans une recherche de filiations ou de parallélismes entre les conceptions occidentales et mésoaméricaines. Cependant, le fait de créer un voisinage insolite entre ces deux recherches, incitant chacun à formuler l'analyse de son propre objet en gardant toujours un œil sur celui de l'autre, avec le souci de lancer autant que possible de fragiles passerelles dans sa direction (fût-ce pour mesurer des abymes de différences) s'est avéré une expérience suffisamment féconde pour justifier la présente juxtaposition de ces deux textes.

1 «Pretender la comprensión histórica del pensamiento de una sociedad dada sin haber precisado al menos los rasgos generales de las entidades anímicas, es bordar en el vacio ». C'est la lecture de deux ouvrages admirables, Cuerpo humano e ideología de Alfredo López Austin (dont est tirée cette citation liminale) et Una etnografia de las aimas tzeltales de 
Pedro Pitarch Ramón ${ }^{1}$, qui m'a stimulé à tenter de présenter synthétiquement les enjeux de la représentation de la personne, et plus précisément de la dualité âme/corps, dans l'Occident médiéval. Sans doute la comparaison avec les conceptions des anciens nahuas ou des actuels tzeltals repousse-t-elle la vision chrétienne au loin, à la mesure de l'opposition obligée entre polythéisme et monothéisme, qui semble justement trouver dans la conception de la personne l'un de ses aspects les plus irréductibles². L'essentiel est moins ici le nombre des entités animiques et corporelles admises par les différentes cultures, que la recherche d'une rupture ou au contraire d'un entrelacement entre le somatique et le psychique, l'individuel et le collectif, l'ordre biologique et l'ordre social, l'humain et le divin.

2 Le christianisme - dont on ne connaît pas d'essence intemporelle, mais seulement des incarnations socio-historiques successives - est, au moins dans sa phase médiévale, un monothéisme pour le moins complexe, de sorte que le fonctionnement du couple âme/ corps s'y révèle moins simple qu'il n'y paraît. On veut en particulier souligner la nécessité de distinguer la conception duelle du christianisme médiéval (qui distingue en effet deux entités fondamentales: l'âme et le corps) et le dualisme auquel, sous les espèces du manichéisme puis du catharisme, le christianisme s'est effectivement affronté et dont il a toujours cherché à se démarquer (dualisme s'entendra ici comme affirmation d'une incompatibilité entre le charnel et le spirituel, conduisant à une dévalorisation complète du matériel, et n'accordant de positivité qu'à un spirituel entièrement séparé).

3 C'est donc dans un entre-deux qu'il faut situer les conceptions de la personne dans l'Occident médiéval : entre la séparation absolue du dualisme manichéen et la fluidité des entités multiples des polythéismes. Il s'agira donc ici d'explorer les conceptions médiévales de la dualité corps/âme, en tenant compte, autant qu'il est possible en un espace réduit, de la diversité des représentations et de leurs évolutions au cours du millénaire médiéval, et en retenant trois questions principales. D’une part, la personne chrétienne est-elle bien conçue au Moyen Âge comme une simple dualité corps/âme - et sinon, quel statut donner aux éléments qui viennent compliquer ce tête-à-tête ? D'autre part, y a-t-il entre âme et corps une véritable disjonction, voire une incompatibilité fondamentale - ou au contraire recherche-t-on entre eux des modalités d'association et d'interrelation? Enfin, on se demandera quelle est la signification sociale de ces formulations de la relation âme/corps - et on proposera de voir en elles une matrice idéologique fondamentale de la société médiévale occidentale.

Ce faisant, on délaissera quelque peu une approche plus classique des conceptions chrétiennes de la personne, dans lesquelles M. Mauss voyait déjà « la définition la plus achevée » de la catégorie du moi, et où l'historiographie récente s'est plu à décrire la naissance de l'individu, les origines de l'individualisme ou encore l'affirmation d'une subjectivité et d'une intimité psychologique ${ }^{3}$. Sans nier la pertinence de cette démarche, ni les relations qu'elle entretient avec les aspects traités ici, on voudrait moins revenir sur ce processus de l'individuation chrétienne que s'engager dans une autre voie, davantage délaissée, qui considère l'âme comme un principe social. Non certes exactement à la manière de Durkheim, pour lequel l'âme n'est rien d'autre que l'identité collective du groupe incarnée dans l'individu ${ }^{4}$; mais simplement parce qu'on croit pouvoir constater que les énoncés relatifs à la personne, en dépit de leur autonomie relative, mettent en jeu des homologies partielles et des interactions réciproques avec des aspects importants des structures sociales. Dans le cas de l'Occident médiéval, une telle analyse doit prendre pour objet principal le complexe rapport corps/âme, et le recours 
aux énoncés théologiques est ici obligé. En effet, pour l'historien médiéviste, la théologie apparaît comme le lieu où se formulent les principaux fondements idéologiques d'une société structurée par l'Église - ce qui toutefois ne signifie nullement que la théologie se ramène à un corps de dogmes unifiés et immuables. Enfin, il convient de faire place à d'autres formes d'expression, textuelles ou visuelles, qui permettent d'évaluer l'étendue et les limites de l'emprise ecclésiale.

\section{Représentations médiévales de la personne, entre dualité et ternarité}

5 La représentation duelle de la personne humaine - âme et corps - n'est certes pas une innovation du christianisme. Ainsi, déjà se pense la personne dans la tradition platonicienne, qui pèse si fort sur la théologie chrétienne. Dans l'Empire romain, règne un "dualisme bienveillant», mélange de hiérarchie ferme et de bienveillante sollicitude entre l'âme et le corps : tel est le «style de gouvernement » qui prévaut entre eux, selon la belle expression de P. Brown qui invite à différencier subtilement les formes du rapport âme/corps ${ }^{5}$. La théologie médiévale offre des centaines d'occurrences de l'énoncé suivant, qui peut être tenu pour l'axe central de ses conceptions : l'être humain est formé de la conjonction d'un corps, charnel et périssable, et d'une âme, entité spirituelle, incorporelle et immortelle. Soit ce qu'on appellera une conception duelle - mais non nécessairement dualiste. Toutefois, plusieurs aspects compliquent cette assertion.

61 - Le christianisme trouve dans la Bible - dans les conceptions judaïques et chez saint Paul - une représentation ternaire de la personne «esprit, âme, corps» (I Thes 5, 23). L'âme (anima, psyché) est le principe animateur du corps, que possèdent également les animaux, tandis que l'esprit (spiritus, pneuma), donné à l'homme seul, le met en contact avec Dieu; et c'est pourquoi « l'homme spirituel est plus haut que l'homme psychique » (I Cor 15, 40-50). Cette trilogie, reprise par Augustin, parcourt la théologie jusqu'au XII siècle (chez Hugues de Saint-Victor, notamment), au prix d'infinies variations du vocabulaire, qui rendent impossible une présentation synthétique de cette question ${ }^{6}$. De même, Augustin et la tradition qui s'en inspire, notamment au XII ${ }^{e}$ siècle, distinguent dans l'âme trois instances, donnant lieu notamment à trois types de visions: la vision corporelle, qui se forme dans l'âme par l'instrument des yeux corporels; la vision spirituelle, qui concerne les images mentales ou oniriques, à la semblance des corps mais incorporelles; enfin la vision intellectuelle, acte de l'intellect dépourvu de toute semblance corporelle ${ }^{7}$. Quoique cette distinction ne soit pas directement superposable à la trilogie paulinienne - son objet est différent, et elle subordonne spiritus à mens, au lieu de le placer au-dessus d'anima -, elle conduit également à penser un niveau intermédiaire entre la matière et l'intellection, dont l'objet est d'articuler le corporel et le spirituel.

7 La scolastique du XIII ${ }^{\mathrm{e}}$ siècle tend à réfuter ces présentations ternaires (saint Thomas affirmant clairement que esprit et âme sont une seule et même chose ${ }^{8}$ ). Mais, au sein d'une dualité stricte, la diversité antérieurement exprimée trouve cependant sa place, puisque l'âme est réputée dotée de trois facultés: végétative (forme de vie également partagée par les plantes), animale (partagée par les bêtes) et rationnelle (propre à l'homme). Si la théorie d'une coexistence de trois substances animiques parait avoir été professée, la plupart des maîtres optent pour l'idée d'une seule essence animique, dotée de trois puissances. Et pourtant, la dualité de l'âme - d'un côté principe animateur du 
corps, de l'autre entité ayant en elle-même sa propre fin - est encore soulignée par Albert le Grand, avec bien d'autres théologiens de son siècle ${ }^{9}$. Cette présentation rapide suggère que la notion chrétienne de l'âme subsume deux éléments au moins (on néglige les exposés théologiques qui, notamment au $\mathrm{XII}^{\mathrm{e}}$ siècle, distinguent jusqu'à cinq parties de l'âme): d'un côté, le principe de force vitale qui anime le corps (l'âme de Paul, les puissances sensitive et animale des scolastiques, soit ce que les tzeltals de Cancuc nomment " ave del corazon $\left.{ }^{10} »\right)$; de l'autre, l'âme rationnelle qui rapproche l'homme de Dieu. En conséquence, ou bien la théologie dissocie ces deux aspects et tend alors vers une anthropologie ternaire ; ou bien elle les englobe dans une même entité, de sorte que l'âme est un principe double, qui relève du corps charnel qu'elle anime, et en même temps partage avec Dieu ses plus hautes qualités. C'est encore la scolastique du XIII ${ }^{e}$ siècle qui, pensant une âme unique dotée de trois puissances, offre l'une des meilleures résolutions de cette contradiction. Mais il reste que la dualité «ave del corazon/ch'ulel » est sans doute la part des représentations tzeltales la moins irréductible aux conceptions chrétiennes ${ }^{11}$.

82 - L'interface âme/corps préoccupe grandement les théologiens médiévaux. La plupart d'entre eux attribuent à l'âme des puissances sensibles qui lui permettent de parvenir par elle-même et indépendamment $d u$ corps à une connaissance du monde sensible. Toutefois, dans sa radicalité anthropologique, Thomas d'Aquin nie l'existence de telles puissances sensibles, retirant ainsi à l'âme seule toute capacité de contact direct avec l'univers matériel, et rendant plus nécessaire encore son union avec le corps ${ }^{12}$. Il faut noter que dans les théories antérieures, l'attribution à l'âme d'une capacité d'expérience sensible était considérée comme une marque d'éminence et d'indépendance, et non comme une compromission inconvenante avec le monde corporel. Ainsi, selon les conceptions, la fonction d'interface entre le spirituel et le matériel peut relever du corps ou de l'âme.

93 - Si l'on se réfère à la distinction entre entités animiques et centres animiques, introduite par A. López Austin dans Cuerpo humano e ideología, le panorama se complique encore. En ce qui concerne les conceptions médiévales, la question de savoir dans quelles parties du corps se trouve l'âme, pourrait certes être évacuée, au motif que l'âme spirituelle est illocalis, étrangère à toute dimension spatiale, et ne peut donc être contenue dans le corps par mode de localisation. Pourtant, une révolution, discrète mais déterminante, s'opère au cours $\mathrm{du} \mathrm{XII}^{\mathrm{e}}$ siècle, reconnaissant finalement que l'âme est localis, localisable ${ }^{13}$. Mais l'âme n'est pas pour autant contenue de manière simple dans le corps, et l'aristotélisme de Thomas d'Aquin conduit à affirmer que l'âme, plutôt que d'être dans le corps, englobe ce dernier : « les choses spirituelles contiennent ce dans quoi elles sont ${ }^{14} »$. On voit cependant se dégager une dualité de centres animiques. Le cœur, déjà perçu par les premiers ermites du désert d'Égypte comme le centre de la personne, "le point de rencontre entre le corps et l'âme, entre l'humain et le divin ${ }^{15}$ », fait au Moyen Âge l'objet d'une promotion croissante qui assure son «triomphe comme localisation de l'âme ${ }^{16}$ ". Mais la tête, comme siège de l'âme résiste, comme on le constate par exemple chez le poète cistercien du XIV siècle, Guillaume de Diguleville ${ }^{17}$. Encore convient-il d'ajouter que, quoiqu'associée à un centre animique privilégié, tête ou cœur, l'âme se trouve aussi répandue dans tout le corps. Même Thomas d'Aquin, qui retire pourtant à l'âme ses puissances sensibles, insiste sur l'interface inverse: les esprits animaux, ces «vapeurs subtiles par lesquelles les forces de l'âme sont diffusées dans les parties du $\operatorname{corps}^{18} »$. Ainsi s'expliquent toutes les interférences entre l'âme et le corps. 
L'âme habite bien le corps, dans sa totalité et en certains de ses centres privilégiés, tête et cœur, même si par sa nature elle échappe aux limites d'une telle localisation.

4 - On fera encore un ajout, qui pourrait paraitre à la limite du problème traité ici, mais qui se révèle néanmoins éclairant. Dans le monde médiéval, la personne humaine ne se conçoit pas seule - sans même parler des réseaux sociaux dans lesquels elle s'insère - : deux entités sont - du moins à partir du $\mathrm{XI}^{\mathrm{e}}$ siècle - associées de manière personnelle et indéfectible à toute vie chrétienne. Chaque être reçoit en effet, de sa naissance à sa mort, un ange gardien qui veille sur lui, et aussi - on le mentionne moins souvent - un diable personnel qui s'emploie à le tenter ${ }^{19}$. Sans doute ces deux esprits sont-ils extérieurs à la personne, mais ils lui sont si étroitement attachés que les actions de l'individu et sa vie entière seraient réputées incompréhensibles, si l'on ne prenait en compte l'action de ces deux représentants des forces divines et maléfiques. Ange gardien et diable personnel peuvent ainsi être considérés comme des " appendices de la personne chrétienne », dont le rôle dans le processus de l'individuation chrétienne mérite d'être évalué à sa juste mesure. Il pourrait paraître peu rigoureux d'établir ici une comparaison directe avec certains des « labs » de Cancuc, ces esprits à la fois intérieurs et extérieurs à la personne, menaçants ou bienveillants ${ }^{20}$. Mais du moins peut-on suggérer que la personne chrétienne n'est pas totalement dépourvue de prolongements externes, ou plus précisément que les conceptions chrétiennes de la personne se soucient également, à leur manière, de produire une articulation entre intérieur et extérieur, entre les composantes propres de la personne et les entités qui régissent l'univers.

115 - L'origine de l'âme individuelle est longtemps restée une question délicate pour les auteurs chrétiens ${ }^{21}$. Déclarant qu'il s'agit d'un " mystère insoluble », Augustin ne parvient pas à choisir entre les différentes thèses en présence : la théorie d'une préexistence de toutes les âmes, créées d'emblée lors de la Création et s'incarnant ensuite à mesure de la conception des individus; le traducianisme, selon lequel l'âme serait transmise par les parents et formée à partir de leur semence; enfin, le créationisme qui suppose que chaque âme est créée par Dieu lors de la conception de l'enfant, et aussitôt infusée dans l'embryon. Au cours des siècles médiévaux, c'est cette dernière thèse qui s'impose en un processus lent et indécis, et finalement chez les scolastiques des XII et XIII ${ }^{e}$ siècles, le choix est clair en faveur du créationisme. Encore convient-il de préciser, comme le fait Thomas d'Aquin, que l'embryon, dans les premiers jours de sa formation, est animé par une âme végétative, puis par une âme sensitive, qui ne procèdent nullement de Dieu mais d'un développement propre du corps engendré par la force de la semence paternelle; enfin, lorsque l'embryon atteint un degré de formation suffisamment digne, l'âme rationnelle est créée par Dieu et infusée dans l'embryon, où elle remplace l'âme sensitive, tout en intégrant en elle les puissances végétatives et sensitives de cette dernière ${ }^{22}$. On repère donc une triple origine de la personne: le corps, issu de la procréation; l'âme animale produite par la vertu paternelle; l'âme rationnelle créée par Dieu. Toutefois, en l'être achevé, cette triple origine se fond en une dualité essentielle.

12 Une miniature exceptionnelle, réalisée vers 1490 dans l'entourage du duc de Bourgogne, montre la création et l'infusion de l'âme individuelle (fig. ${ }^{23}$ ). Elle établit un partage des tâches clair : le couple humain, surpris dans l'intimité nocturne et dénudée de son lit, est manifestement responsable de l'engendrement du corps de l'enfant à naître. Quant à l'âme, elle descend de plus haut qu'eux; elle est l'œuvre de Dieu, de cette Paternité suprême qui caractérise la divinité trinitaire. L'âme intellectuelle, substance immatérielle et incorporelle, ne saurait en effet être causée par la génération; elle ne peut procéder 
que de Dieu, et les théologiens s'emploient à souligner que rien de l'âme des parents ne se transmet à leurs enfants. Le plus remarquable dans cette image est l'irruption de la transcendance trinitaire dans le décor si prosaïquement modeste et contemporain d'une chambre à coucher - étonnante manière de montrer la mobilisation de la Suprême Divinité et son engagement théophanique à l'origine d'une banale destinée individuelle. À cet égard, on remarque que le phylactère divin porte le verset de Gn 1,26 ( Faciamus hominem ad imaginem et similitudinem nostram ") : c'est bien en effet la décision divine de créer le premier homme qui se trouve actualisée, rejouée quotidiennement, lors de la formation de chaque âme individuelle ${ }^{24}$.

Fig. 1

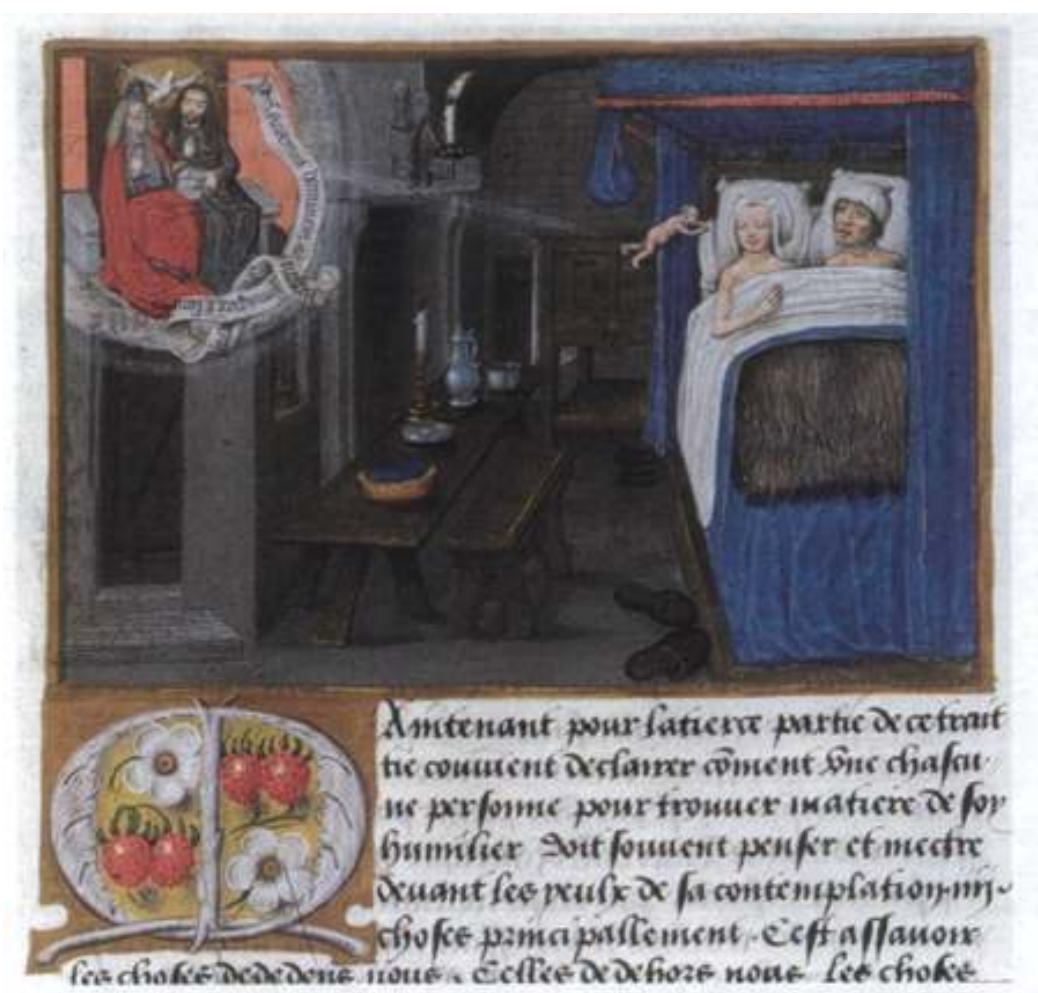

Les parents, la Trinité et l'infusion de l'âme lors de la conception de l'enfant (Paris, Bibliothèque de l'Arsenal, ms. 5206, fol. 174, vers 1490).

Fig. 2 


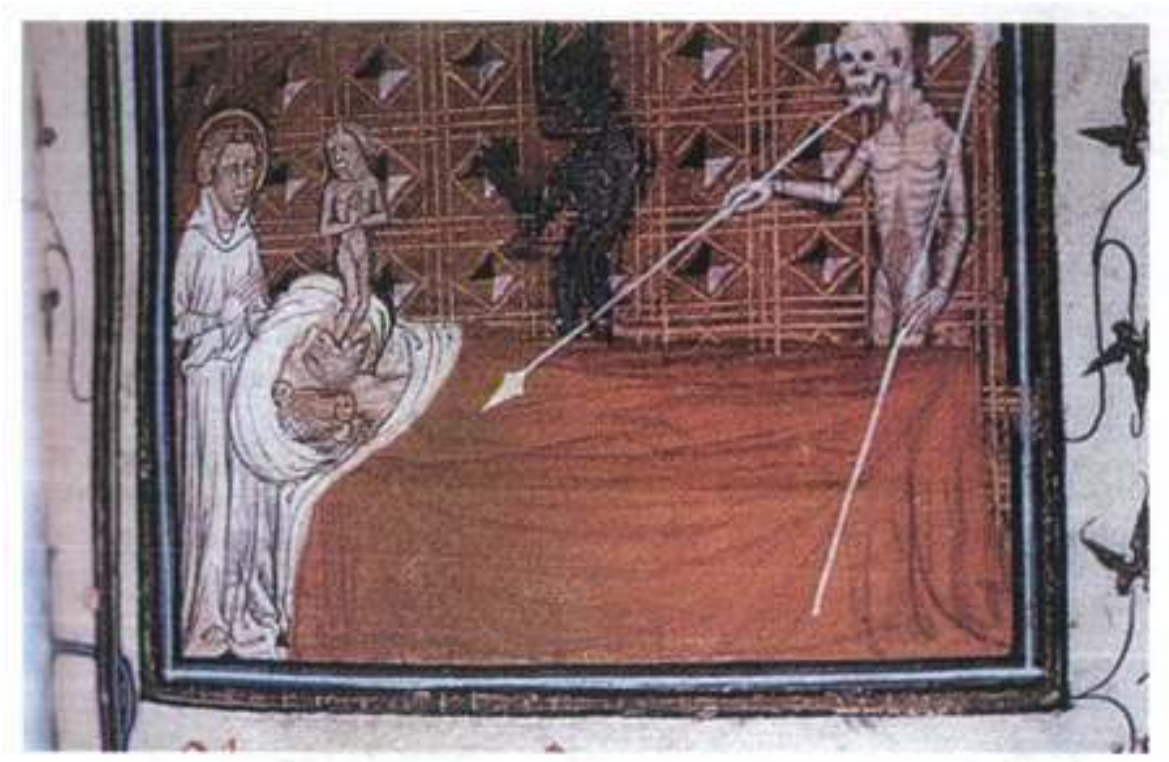

L'âme sortant du corps lors de la mort (miniature de Remiet, Leyde, Bibliothèque Universitaire, BPL 74, fol. 92, Pèlerinage de l'âme, c. 1400-1410).

Le choix du créationisme - que cette miniature illustre avec éclat - est riche de sens. Il assure une cohérence au processus de formation de la personne, en associant étroitement la création de l'âme au processus de la reproduction charnelle - mais sans toutefois établir, pour celle-là, le moindre lien avec les parents, comme le supposait le traducianisme. D'autre part, contrairement à la théorie de la préexistence des âmes, le créationisme singularise le destin de chaque âme, désormais lié à la conception propre des êtres individuels. Il est donc un aspect de l'individuation de la personne chrétienne, accompli à travers un rapport à Dieu dont cette miniature suggère combien il est écrasant.

6 - Si la conception suppose la conjonction d'une âme et d'un corps, la mort chrétienne se présente - et se représente - comme la séparation de ces deux principes (fig. 2). Exemple parmi bien d'autres possibles, une miniature du début du $\mathrm{XV}^{\mathrm{e}}$ siècle montre l'âme comme une figurine nue, sortant de la bouche du mort, en son ultime souffle : image inversée de l'accouchement, puisque mourir chrétiennement, c'est naître à la vie éternelle. Il est impossible de développer ici l'analyse des conceptions médiévales de l'au-delà, en sa redoutable dualité - enfer et paradis -, partiellement tempérée par la naissance du purgatoire au XII ${ }^{\mathrm{e}}$ siècle ${ }^{25}$. On soulignera seulement deux aspects touchant directement à la conception même de l'âme. On note d'abord un effort des clercs pour localiser les âmes, pour leur conférer un lieu délimité et connu, comme l'est justement le purgatoire : tout en développant ainsi une logique d'encadrement spatial qui est au principe même de la société féodale, ils s'emploient à mieux séparer les vivants des morts - ou plus exactement de cette partie vivante des morts que sont les âmes -, s'opposant ainsi aux conceptions folkloriques de l'errance des morts, ou encore à l'invasion des revenants qui, comme l'a montré Jean-Claude Schmitt, résistent à un tel enfermement ${ }^{26}$.

En second lieu, on soulignera que les conceptions de l'âme doivent être mises en relation avec l'importance que le christianisme médiéval confère à l'au-delà, conçu comme le lieu fondamental où, par-delà la confusion du monde terrestre, se réalise la Justice divine. L'au-delà offre l'horizon d'une société enfin ordonnée, donnant sens aux péripéties et aux 
injustices de l'ici-bas. Il est aussi le miroir où chaque être doit lire sa Vérité ultime, sous les espèces des châtiments éternels ou des béatitudes célestes. Dès lors que toute destinée humaine doit se mesurer et se penser à l'aune de cette rétribution post mortem, le christianisme ne peut se satisfaire d'une immortalité impersonnelle, qui caractérise notamment le monde des morts de la Grèce antique ${ }^{27}$, ni accepter que la mort désagrège, même partiellement, les entités composant la personne, comme on le constate dans les conceptions nahuas ou tzeltales ${ }^{28}$. Les représentations chrétiennes doivent assurer au contraire, par-delà la mort, une forte continuité de la personne, de sorte que la rétribution dans l'au-delà s'applique bien à l'être qui, ici-bas, en a mérité les rigueurs ou les joies. On verra plus loin ce qu'il en est à cet égard du corps; mais cela suppose pour le moins une unité de l'âme, et surtout une identité aussi proche que possible de celle de l'homme qu'elle vivifiait. Comme on le verra, le christianisme médiéval pousse loin cette identification de l'âme et de la personne - et pas seulement parce qu'il suit largement une tradition néoplatonicienne pour laquelle l'homme, c'est son âme. Il faut toutefois noter quelques résistances à l'individualisation de l'âme. Ainsi, comme l'explique le moine Guibert de Nogent, au XII ${ }^{e}$ siècle, l'âme dans l'autre monde ne peut être désignée par son nom. On la reconnaît certes - elle ne disparaît donc pas dans l'anonymat des morts -, mais elle a perdu un aspect fondamental de son identité singulière - car elle appartient désormais à la communauté élargie des morts, au sein de laquelle tous doivent pouvoir éprouver une interconnaissance généralisée ${ }^{29}$. Les conceptions médiévales oscillent donc dans un entre-deux: l'âme séparée n'est ni un vague spectre impersonnel, ni une personne au sens plein du terme.

16 Synthétisant cette première partie, on constate que les conceptions médiévales ne se réduisent pas à une dualité simple (présentant de ce fait plus de proximité avec les conceptions non-chrétiennes qu'on ne pourrait le croire). On y repère en particulier une tension entre une représentation duelle et une représentation ternaire de la personne. L'enjeu en est notamment le statut accordé au principe de force vitale (spirituel, mais destiné à l'animation du corps), ainsi qu'aux nécessaires médiations entre le matériel et le spirituel (images mentales des choses corporelles chez Augustin, ou encore puissances sensibles de l'âme). Ces instances peuvent s'autonomiser, suggérant ainsi une tripartition de la personne, ou bien être englobées dans la notion d'âme, ce qui confère souvent à celle-ci de notables affinités avec le monde matériel. Surtout, l'évolution des conceptions médiévales fait apparaître un glissement de la ternarité vers des formulations plus binaires. Il faut donc à la fois souligner la complexité de la personne chrétienne et reconnaître qu'un processus historique tend à subsumer cette complexité au sein d'une structure duelle. En bref, si l'énoncé de la dualité âme/corps ne suffit certainement pas à rendre compte de la personne chrétienne, il en définit néanmoins la structure fondamentale, comme le soulignent les représentations visuelles de la procréation et de la mort.

\section{Entrelacer le corps et l'âme}

17 Définir la personne comme une dualité corps/âme est insuffisant, outre pour les raisons déjà invoquées, parce qu'un tel énoncé ne dit rien du «style de gouvernement » établi entre ses deux composantes - relation pourtant plus importante que les termes qui la composent. On sait que la tradition platonicienne, reprise par de nombreux auteurs du Haut Moyen Âge, tels Boèce ou Grégoire le Grand, identifie l'homme à son âme et 
considère le corps comme un vêtement transitoire et "superflu", un instrument au service de l'âme et extérieur à elle, ou encore comme une prison qui entrave le libre essor de l'esprit. Pourtant, la dynamique des conceptions anthropologiques médiévales apparaît plutôt comme un dépassement du dualisme néoplatonicien. Amorcée par Augustin, qui finit par récuser la définition de l'âme comme prison du corps ${ }^{30}$, cette dynamique donne lieu au XII ${ }^{e}$ siècle à de magnifiques formulations. Voici comment la savante abbesse Hildegarde de Bingen décrit l'infusion de l'âme :

«le vent vivant qu'est l'âme entre dans l'embryon (...), le fortifie et se répand en toutes ses parties, comme un ver qui tisse sa soie: il s'y installe et s'y enferme comme dans une maison. (...) il emplit de son souffle tout cet assemblage, de même qu'une maison tout entière est illuminée par le feu qu'on y fait (...) ; l'âme garde la chair, grâce au flux du sang, dans une humidité permanente, de même que les aliments, grâce au feu, cuisent dans la marmite; elle fortifie les os et les fixe dans les chairs, de façon que ces chairs ne s'effondrent pas : tout comme un homme bâtit sa maison avec du bois pour qu'elle ne soit pas détruite ${ }^{31}$ ».

Outre la saisissante qualité concrète des métaphores appliquées à l'âme, on constate que celle-ci ne descend pas dans une sinistre prison, mais dans une maison qu'elle se réjouit d'habiter, d'autant plus qu'elle l'a construite et surtout conformée selon ses exigences et par sa vertu propre. En conséquence, l'attachement du corps et de l'âme est un fait positif, voulu par Dieu et haï par Satan ${ }^{32}$.

19 Le caractère positif de ce lien est également exprimé par les maîtres en théologie des XII et XIII ${ }^{\mathrm{e}}$ siècles qui, loin de souligner seulement l'étrange compagnie "de l'esprit vivificateur et de la glaise terrestre ${ }^{33}$ ", indiquent que le corps a été créé en commensurabilité avec l'âme, et que celle-ci est dotée d'une unibilitas, c'est-à-dire d'une aptitude à s'unir au corps. Pour l'évêque de Paris, Pierre Lombard, qui ne passe pourtant pas pour un grand progressiste, le statut de la personne humaine montre que « Dieu a le pouvoir de conjoindre les natures disparates de l'âme et du corps pour réaliser un assemblage unifié par une profonde amitié ${ }^{34} »$. Ce qui définit l'homme, ce n'est ni l'âme, ni le corps, mais l'existence d'un composé unifié, formé de ces deux substances. Quant au thème de l'amitié entre le corps et l'âme, il ne fait que s'amplifier, dans la littérature morale où le genre des Débats du corps et de l'âme souligne la tristesse qu'ils ont à se séparer ${ }^{35}$, tout comme dans la spéculation théologique où, au milieu du XIII ${ }^{\mathrm{e}}$ siècle, Bonaventure analyse le désir réciproque que l'âme et le corps ont de s'épouser ${ }^{36}$.

20 L'aristotélisme permet à Thomas d'Aquin de pousser cette dynamique à son point extrême. Conformément à l'hylémorphisme du philosophe, l'homme n'est plus pensé comme l'union de deux substances: l'âme n'est plus une entité autonome associée au corps, mais la forme substantielle du corps, qui lui permet d'exister sur un mode individué. L'interdépendance de l'âme-forme et du corps-matière est totale, comme le souligne M. D. Chenu : « contre tout dualisme, l'homme est constitué d'un seul être, où la matière et l'esprit sont les principes consubstantiels d'une totalité déterminée, sans solution de continuité, par leur mutuelle inhérence : non pas deux choses, non pas une âme ayant un corps ou mouvant un corps, mais une âme-incarnée et un corps-animé, de telle sorte que l'âme est déterminée, comme "forme » du corps, jusqu'au plus intime d'elle-même, à ce point que sans corps, il lui serait impossible de prendre conscience d'elle-même ${ }^{37}$ ». Et Thomas ne se contente pas d'affirmer, comme nombre de ses prédécesseurs, que l'union avec le corps est, pour l'âme, naturelle et bénéfique, il va jusqu'à dévaloriser radicalement l'état de l'âme séparée, puisque le corps est nécessaire non seulement à la plénitude de la personne humaine, mais aussi à la perfection de l'âme 
elle-même, incapable sans lui d'accomplir pleinement ses facultés cognitives ${ }^{38}$. Jugeant l'état de l'âme séparée contra naturam, Thomas affirme pour la première fois que l'union avec le corps peut seule conférer à l'âme sa perfection et sa pleine ressemblance avec Dieu.

Il convient ici de souligner un double aspect de la démarche thomiste. Elle formule de manière aussi tranchée que possible la dualité du corps et de l'âme, dont elle distingue radicalement les natures respectives, en éliminant tout mélange ou point de contact, tel que les puissances sensibles de l'âme. Mais l'accentuation de cette dualité ne vise qu'à mieux dépasser le dualisme, en reconnaissant au corps un statut super-éminent et en accordant à l'unité corps-âme la plus nette positivité. C'est dans la mesure même où l'âme et le corps sont davantage distingués quant à leurs natures, que leur interdépendance s'accroît et que leur couplage s'avère plus essentiel. L'importance accordée ici au thomisme rend nécessaire une remarque de méthode : la perspective de l'historien ne saurait être celle du spécialiste de la théologie qui, surtout s'il est dominicain, tend souvent à isoler Thomas d'Aquin dans la singularité de sa puissance de pensée ${ }^{39}$. Sans nier ni cette force intellectuelle, ni sa radicalité, l'historien est davantage enclin à voir en son œuvre la pointe extrême d'une dynamique intellectuelle et sociale qui traverse les siècles centraux du Moyen Âge. Il ne s'agit certes pas d'y voir la doctrine officielle de son temps d'autant que la condamnation de 1277, qui la vise en partie, montre que cette pensée outrepasse la capacité de réception de l'institution ecclésiale -, mais du moins peut-on admettre qu'elle vaut non seulement pour elle-même, mais aussi pour sa capacité à révéler cette dynamique avec éclat.

22 Ainsi, l'âme séparée, en son imperfection, désire son corps, et s'impatiente des retrouvailles que l'eschatologie chrétienne lui promet, en prélude au Jugement dernier. Il faut en effet évoquer ici la résurrection des corps, cette croyance fondamentale à laquelle C. Bynum a récemment accordé une attention méritée ${ }^{40}$, car c'est sans doute l'un des aspects les plus originaux (et les plus délicats à faire admettre ${ }^{41}$ ) du christianisme auquel, de surcroit, la société médiévale a conféré une importance toute particulière. Fondée sur l'Évangile, mentionnée dans le Credo et défendue par tous les théologiens médiévaux, la doctrine de la résurrection générale des corps, destinés à être réunis aux âmes lors du Jugement dernier, ne souffre aucune contestation (sinon parmi les hérétiques radicaux, comme les cathares). On doit cependant mentionner un conflit entre une conception spirituelle des corps ressuscités et une interprétation matérielle. Se prévalant de saint Paul qui évoque la résurrection d'un « corps spirituel » et affirme que « la chair et le sang ne peuvent hériter du Royaume » (I Cor 15), des auteurs comme Origène ou Grégoire de Nysse conçoivent pour les ressuscités un corps éthéré, semblable à celui des anges, sans âge et sans sexe. Mais toute la tradition médiévale, dans le sillage d'Augustin, admet au contraire la pleine matérialité des corps ressuscités : la chair qui ressuscite est bien celle des corps terrestres individuels, reformés avec tous leurs membres, y compris les organes sexuels et digestifs dont les spiritualistes voulaient les débarrasser (fig. 3). Il s'ensuit une obsession quasi-maniaque de l'intégrité des corps ressuscités, auquel pas un grain de poussière ne doit manquer et qui, en cas de mutilation ou de dévoration par les animaux, doivent être entièrement reformés. Cette exigence conduit un théologien aussi sérieux qu'Augustin à démontrer que la matière de tous les ongles et de tous les cheveux coupés au cours de la vie doit être également réunie au corps ressuscité (mais sous une forme transformée, car sinon elle produirait une effrayante laideur ${ }^{42}$ ). Cette conception peut nous apparaitre cocasse, mais elle ne surprendrait guère les tzotzils de Chenalhó, où la 
coutume voulait que chacun conserve dans un sac tous les ongles et les cheveux coupés depuis sa naissance (ici, non pour le bénéfice d'un improbable corps ressuscité, mais pour éviter à l'âme du mort de devoir rechercher partout ces parties du corps, de sorte que dans cette pratique - dérivée de la croyance chrétienne ? - c'est l'âme qui se soucie des excroissances du corps qu'elle a abandonnét3).

Fig. 3

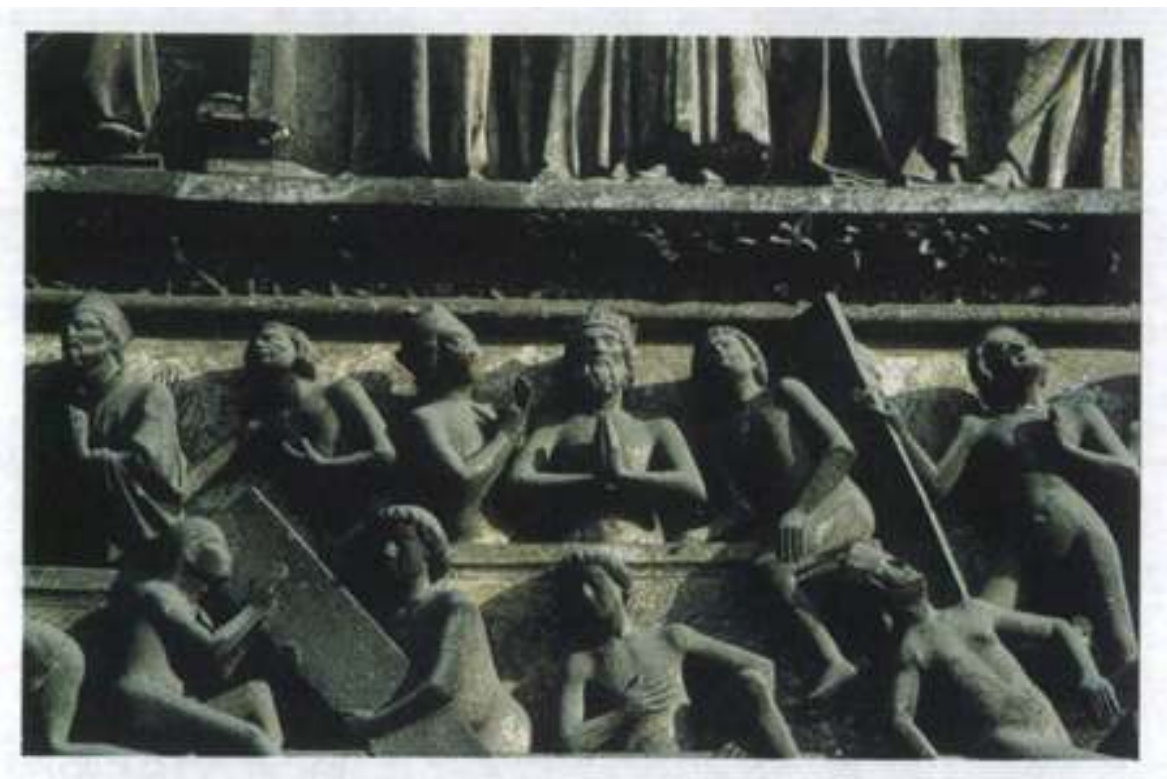

La résurrection des corps lors du Jugement dernier (Cathédrale de Bourges, portail central, vers 1240).

Dans la conception matérielle de la résurrection, la définition du corps des élus comme « corps spirituel» (I Cor 15) ne peut être comprise que comme un oxymore, dont il convient de reconnaitre pleinement le caractère contradictoire : loin de se transformer en esprit, le corps ressuscité conserve la pleine matérialité de sa chair; mais en même temps, il est dit spirituel, parce qu'il acquiert des qualités supplémentaires qui sont normalement celles de l'âme ${ }^{44}$. En premier lieu, le corps glorieux, tout comme l'âme, est immortel et impassible, échappant aux effets du temps et à la corruption. Les traités que les théologiens consacrent aux béatitudes du corps des élus soulignent également sa parfaite beauté, éternellement conservée dans la force de l'âge et dotée de proportions harmonieuses; et le XIII ${ }^{e}$ siècle y ajoute la claritas qui en fait un corps lumineux comme le soleil, voire transparent comme le cristal ${ }^{45}$. Ce corps est également doué de liberté et d'agilité : il a le pouvoir de faire tout ce qu'il veut et de se déplacer comme il l'entend, sans le moindre effort et aussi rapidement que les anges. Pour les clercs médiévaux, le monde céleste n'est pas cet ordre immobile et figé que l'on imaginerait volontiers, et le mouvement est tenu pour une qualité qui sied à la perfection des corps. Enfin, le corps glorieux éprouve une voluptas, qui résulte de l'exercice des cinq sens et se manifeste en chacun de ses membres : s'il est inutile de préciser les limitations que les clercs fixent à la sensualité paradisiaque, du moins la reconnaissance d'une activité céleste des sens souligne-t-elle leur participation nécessaire à la perfection de la personne humaine. Le christianisme médiéval pousse donc très loin la rédemption du corps, jugée nécessaire à la pleine béatitude du paradis (ce « lieu des délices avec les saints », comme disaient les 
dominicains du $\mathrm{XVI}^{e}$ siècle, chargés d'évangéliser les tzeltals ${ }^{46}$ ). Dans sa matérialité charnelle et la complétude de ses membres, le corps, avec ses vertus de beauté, de force, de mouvement et de sensualité, trouve droit de cité dans la société parfaite de Dieu. Cette réhabilitation du corps suppose néanmoins deux exclusions : le corps glorieux est certes un corps complet (donc sexué), mais il est un corps non-sexuel et non-alimentaire, écartant ainsi deux fonctions qui renvoient l'homme à l'éphémère de sa condition mortelle et par conséquent à sa nécessaire reproduction, et que les clercs jugent incompatibles avec le contrôle que le principe spirituel doit exercer sur le corps glorieux. La cuisine et le sexe n'ont de place qu'en enfer.

Avant de prolonger l'analyse du corps glorieux, il convient de mentionner un autre rapport. Car comme l'indique P. Brown, « le rapport âme/corps est une synecdoque pour désigner l'humanité vulnérable face à $\operatorname{Dieu}^{47} »$. Parmi de multiples attestations médiévales de l'équivalence des rapports corps/âme et homme/Dieu, on citera à nouveau Hildegarde de Bingen : à la fin des temps, « Dieu et l'homme ne font qu'un, comme l'âme et le corps, parce que Dieu a fait l'homme à son image et à sa ressemblance ${ }^{48}$ ». À l'image de l'unité glorieuse des corps spirituels, les élus admis dans la société céleste sont rassemblés en Dieu, en un rapport de ressemblance finalement restauré après la rupture produite par le péché originel. La récompense céleste, vision béatifique de Dieu, c'est-à-dire parfaite compréhension de l'essence divine, suppose une union totale en Dieu, dont les théologiens reconnaissent qu'elle tend à une quasi-divinisation de l'homme ${ }^{49}$. Il est notable que ces conceptions de la béatitude céleste ont particulièrement choqué les païens de l'Empire romain : l'assomption de l'humain jusqu'au monde divin, jointe à la glorification des corps élus partageant désormais le sur-corps autrefois réservé aux maîtres de l'olympe $\mathrm{e}^{50}$, leur apparaissaient - au même titre que l'Incarnation de Dieu comme autant de mélanges scandaleux entre l'humain et le divin. Les relations entre le corps et l'âme d'une part, entre l'humain et le divin d'autre part, constituent bien deux aspects strictement corrélés de l'anthropologie chrétienne.

\section{L'articulation corporel/spirituel comme modèle social}

Loin de revendiquer leur séparation comme un idéal, le corps glorieux propose à la chrétienté médiévale l'horizon d'une articulation entre le corps et l'âme. Mais encore faut-il préciser que cette relation est fondamentalement hiérarchique - et ici je me sépare des analyses de C. Bynum ${ }^{51}$. Car le corps glorieux se caractérise par son obéissance absolue aux volontés de l'âme; et s'il est dit spirituel, c'est parce qu'il est entièrement soumis à l'âme. Bonaventure, évoquant le désir mutuel que l'âme et le corps ont de se réunir, nous prémunit contre toute idée d'une union égalitaire, en précisant l'existence d'un «ordre de gouvernement" par lequel le corps obéit entièrement à l'âme ${ }^{52}$. Il ne saurait y avoir de rédemption du corps qu'au prix de sa totale servilité, selon une dialectique bien chrétienne de l'abaissement et de la glorification. Le corps glorieux est donc, paradoxalement, un modèle de la souveraineté de l'âme, de la domination de l'âme sur le corps - et c'est dans ce cadre seul que prend sens l'insistance sur l'aspect charnel de la résurrection. Il invite à penser une relation entre le corporel et le spirituel, qui ne soit ni un mélange ou un état intermédiaire (pas de syncrétisme, donc!), ni une totale disjonction (qui reconduirait au dualisme). Le "corps spirituel» se définit au contraire comme une union des deux principes au sein d'une même entité - mais encore faut-il préciser qu'il s'agit d'une union hiérarchique (l'âme domine le corps) et dynamique (par 
cette soumission, le corps s'élève, devient conforme à l'âme et doté de qualités merveilleuses ; schéma 1).

Ce modèle est un puissant outil de représentation sociale, dans un monde où le clergé défini justement par son caractère spirituel - assume une position dominante. Dans la société médiévale, la relation spirituel/corporel est à l'image de la relation entre clercs et laïcs ${ }^{53}$. Selon un système de représentation auquel la Réforme grégorienne des $\mathrm{XI}^{\mathrm{e}}-\mathrm{XII}^{\mathrm{e}}$ siècles donne sa plus extrême rigueur, les clercs se définissent par leur rejet de la parenté charnelle et le renoncement proclamé à toute sexualité. Laissant aux laïcs la tâche de reproduire corporellement la société, ils s'intègrent à une institution structurée par la parenté spirituelle, et se consacrent à la reproduction spirituelle de la chrétienté, à travers l'administration des sacrements. Le partage des tâches est net, de sorte que le gouvernement de l'esprit sur le corps apparaît comme le modèle de l'autorité des clercs sur les laïcs, donnée comme moyen de la rédemption de tous. Car la société dans son ensemble ne peut atteindre le salut qu'à la condition de se laisser guider par sa part spirituelle, par un clergé rendu spirituel par son renoncement aux liens de la chair.

Encore faut-il, pour que le corps glorieux fonctionne comme modèle social, qu'il n'y ait pas seulement hiérarchie, mais aussi unité. L'Église, institution dominante du monde féodal, assume d'autant mieux cette unité qu'elle est elle-même un "corps spirituel » (corpus Christi, corpus mysticum). L'Église médiévale, institution incarnée dans le sol de ses immenses possessions, engagée pleinement dans l'organisation de la société des hommes, et dotée d'une matérialité ornementalisée dont la richesse éclate aux yeux de tous les fidèles, ne trouve pourtant de légitimité que par le principe spirituel qui la gouverne et au nom duquel elle gouverne les âmes et les corps. Certes, l'Église n'est pas une entité homogène et elle est divisée par des hiérarchies internes, travaillée par des divergences d'intérêts multiples, et animée par des débats intellectuels parfois violents. Mais, tout en étant plurielle, elle existe institutionnellement, idéologiquement et liturgiquement, comme unité, de sorte qu'on peut bien la définir comme un corps spirituel, ordonnant la matière du monde vers ses fins spirituelles et célestes. Pensée à l'image du corps de la Vierge Marie, l'Église est la Mère virginale qui, par la vertu de l'Esprit, reproduit le corps social tout en revendiquant sa parfaite pureté.

Schéma 1

a)

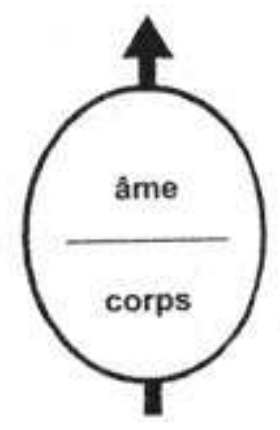

b)

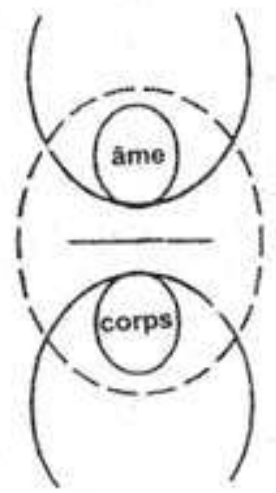

a) Le corps glorieux, modèle idéal de la personne chrétienne.

b) La conception dualiste de la personne. 
Bien qu'elle ne cesse de les opposer, l'Église médiévale cherche surtout à articuler le corporel et le spirituel, à l'image du corps glorieux, mais aussi conformément à la logique de l'Incarnation par laquelle le Fils divin prend chair d'homme, et qui constitue un autre modèle essentiel pour l'Église. La question centrale que soulève son rôle dans la société est : comment faire du spirituel avec du charnel? comment spiritualiser le corporel ? Les sacrements, à commencer par le baptême, n'ont pas d'autre objet. L'évolution du mariage, qui au terme d'un processus séculaire de revalorisation, devient justement au XII ${ }^{\mathrm{e}}$ siècle un sacrement, montre qu'il ne s'agit nullement d'abandonner les laïcs à la chair et au péché : le mariage, sacramentalisé et peu à peu cléricalisé, définit le cadre légitime de l'activité sexuelle et reproductrice et intègre celle-ci au sein d'une alliance de type spirituel, conçue à l'égal de l'union du Christ et de l'Église ${ }^{54}$. Le sacrement du mariage vise ainsi à produire une spiritualisation du lien charnel.

Inutile de multiplier les exemples: qu'il s'agisse du statut du clergé, du mariage, ou encore des images - rendues légitimes parce qu'elles permettent de s'élever de l'objet matériel jusqu'au prototype céleste -, le schéma est le même, toujours fondé sur une double relation de distinction hiérarchique et d'articulation dynamique entre le matériel et le spirituel (schéma 2). L'unité de ces structures est clairement attestée par le fait qu'elles sont toutes également mises en cause par les hérésies qui attaquent la domination de l'Église catholique, notamment entre $\mathrm{XI}^{\mathrm{e}}$ et $\mathrm{XIII}^{\mathrm{e}}$ siècles. Le dualisme cathare, qui entend séparer totalement le spirituel et le matériel, constitue l'expression la plus radicale de cette contestation : il s'en prend à un clergé compromis avec le monde et englué dans sa richesse matérielle, condamne parmi bien d'autres pratiques le mariage et les images - et récuse dans le même mouvement la résurrection des corps. Affirmer que l'esprit ne se sauve que séparé, et que toute alliance avec la matière est nécessairement une corruption, revient à saper les fondements de l'institution ecclésiale et de la société médiévale dans son ensemble. Au contraire, renforçant sa propre logique à travers sa lutte victorieuse contre les hérésies, l'Église n'en apparaît que plus clairement comme une immense machine à spiritualiser le corporel, à entraîner le monde terrestre vers sa fin spirituelle. Réalisation d'une relation parfaite entre le corps et l'âme, le «corps spirituel», tel qu'il s'affirme dans les conceptions matérielles de la résurrection, constitue ainsi une matrice idéologique et sociale, fondamentale dans l'Occident médiéval, puisqu'il place à l'horizon de l'idéal eschatologique un modèle qui fonde la légitimité même de l'institution ecclésiale.

31 Mais la domination de l'Église ne s'exerce pas sans conflits (on vient d'évoquer la radicale contestation hérétique), ni sans négociations qui laissent place, autour de l'axe central des conceptions doctrinales de l'Église, à un champ de représentations distinctes mais en partie acceptables. La place manque ici pour évoquer les conceptions folkloriques qui font de l'âme un double doté d'une réalité très physique ${ }^{55}$. Dans cet univers, ce qui concerne l'âme doit s'incarner dans des gestes ou des faits matériellement constatables: ainsi, lorsque l'on retire une tuile du toit de la maison d'un mourant pour faciliter le départ de son âme ${ }^{56}$, ou encore lorsque l'apparition d'un revenant laisse une trace physique sur le corps du visionnaire. La nécessité de justifier rituels et croyances contribue à conférer aux êtres spirituels une certaine corporéité. Ainsi, les habitants de Montaillou, qui partagent l'idée d'une errance des âmes au milieu des vivants, recommandent à ces derniers de ne pas marcher les bras écartés, afin de ne pas « jeter à terre » quelque esprit en peine ${ }^{57}$. Au reste, la corporéité de l'âme n'est pas totalement étrangère à la pensée de 
l'Église, mais elle a surtout été professée par Tertullien au II siècle et par quelques clercs des $\mathrm{V}^{\mathrm{e}}$-VII ${ }^{\mathrm{e}}$ siècles, pour être ensuite démentie.

Schéma 2
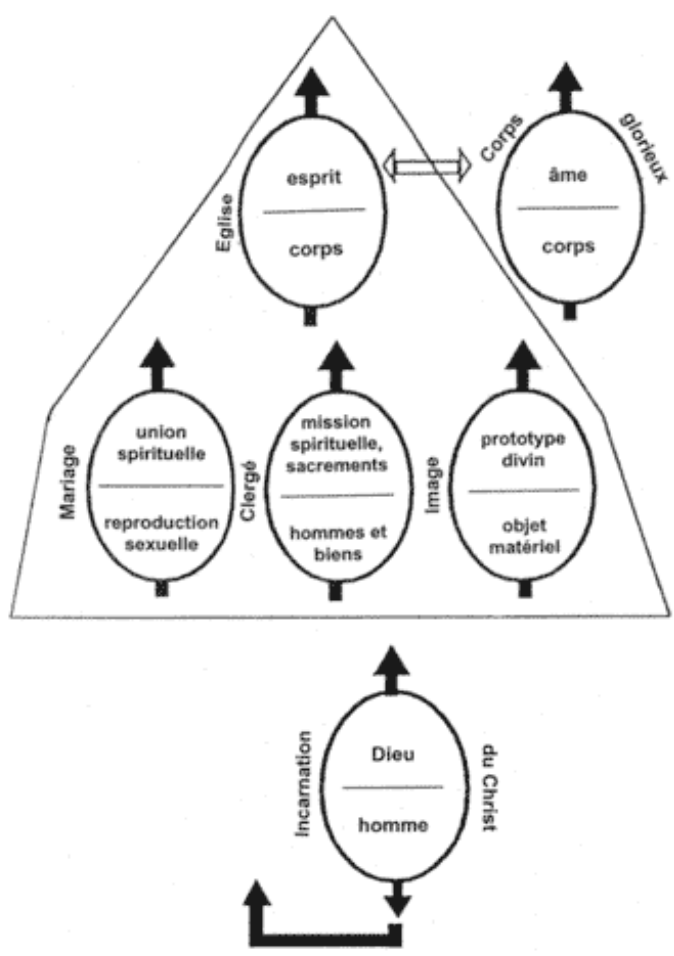

Homologies entre le corps glorieux, l'Incarnation du Christ et la structure ecclésiale.

Pourtant, une tendance à mêler les instances spirituelle et corporelle demeure repérable, y compris au sein des représentations admises et transmises par les clercs. L'image anthropomorphe de l'âme offre une occasion propice pour s'interroger sur ce point. Certes, à proprement parler, l'image ne dit rien de la substance de l'âme, et l'on peut admettre qu'elle montre, conformément à la définition augustinienne de l'âme, une réalité spirituelle dotée d'une similitude corporelle. En ce sens, Guibert de Nogent a raison de se gausser de ceux qui croient que l'âme possède un corps sous prétexte qu'on la représente comme un petit enfant $\mathrm{nu}^{58}$. Pourtant, l'art des $\mathrm{XIV}^{\mathrm{e}}-\mathrm{XV}^{\mathrm{e}}$ siècles accentue la difficulté, en autorisant un véritable portrait de l'âme, double parfaitement individualisé du corps qu'elle habite. Dans le cas de Judas (fig. 4), le portrait n'est guère flatteur : l'âme est incontestablement juive, et son caractère sexué (le sexe des âmes!) souligne la bassesse charnelle du traitre.

S'agissant d'un saint aussi glorieux que Thomas d'Aquin, la corporéité de l'âme ne peut que revêtir une signification bien différente (fig. 5). Il n'est pas déplaisant, après avoir glorifié la pensée thomisme, de montrer ce grand intellectuel sous un tout autre angle. Dans les fresques de Loreto Aprutino, vers 1420, l'ultime scène du cycle consacrée au saint montre, après les funérailles, son âme séparée du corps, mais dotée d'une corporéité remarquablement rebondie et qui, au lieu de s'élever d'elle-même comme un corps aérien, doit faire l'objet d'une étonnante manipulation physique : il lui faut l'aide de saint Pierre et de saint Paul, qui lui font la courte-échelle et la poussent aux fesses, pour s'arracher à la pesanteur et se hisser jusqu'à l'étreinte que lui offre le Christ ${ }^{59}$. Cet 
exemple extrême suffira pour suggérer que ce type de représentation peut relever de deux analyses, complémentaires ou contradictoires selon les cas. On y verra d'abord un phénomène de corporalisation du spirituel, qui pourrait bien constituer, avec la dynamique de spiritualisation du corporel précédemment analysée, les deux versants d'un même système. Ayant l'un et l'autre l'Incarnation de Dieu pour ultime fondement, ces deux dynamiques apparemment inverses, visent conjointement à produire un dépassement du dualisme et une articulation de l'âme et du corps. L'apparence corporelle de l'âme peut ainsi annoncer, par un jeu de chiasme, le corps spiritualisé que les élus revêtiront lors de la résurrection. Elle fait aussi écho au corps-relique du saint, dont l'incorruptibilité reflète la perfection de son esprit, et qui était l'instrument privilégié du contact entre les dévots et les puissances célestes. L'image incarnée de l'âme permet ainsi de construire des réseaux d'échanges et de reflets : elle participe pleinement de la logique ecclésiale, qui toujours s'emploie à jouer des correspondances entre spirituel et corporel, et s'avère même parfaitement capable d'exprimer le spirituel à travers le matériel, pourvu que cette condescendance soit finalement justifiée par une dynamique ascensionnelle ${ }^{60}$.

D'autre part, ce n'est sans doute pas un hasard si une telle image du Docteur en théologie de l'Université de Paris apparaît dans une modeste église des montagnes d'Italie centrale. Il s'agit en effet d'une région dominée par les comtes d'Aquino, descendants des parents de Thomas, qui s'emparent de son culte comme d'une affaire de famille. Là, Thomas est moins un savant de l'Église Universelle qu'un être proche, familier, enraciné dans sa terre natale. Il n'est donc pas exclu que le poids des intérêts de la parenté charnelle et d'une récupération territorialisée du saint ait contribué à "alourdir " l'âme de Thomas. L'essentiel reste bien sûr sa puissance spirituelle et son accès au ciel, conditions de l'efficacité des reliques laissées sur terre, de sorte que l'Église ne saurait prendre ombrage de ces formulations, d'autant qu'elle contrôle finalement le culte. Au total, la tendance à incarner le spirituel et à corporaliser les âmes peut être analysée à la fois comme une des composantes de la logique du système ecclésial, et comme le résultat d'une conciliation avec les intérêts et les représentations des laïcs. 
Fig. 4

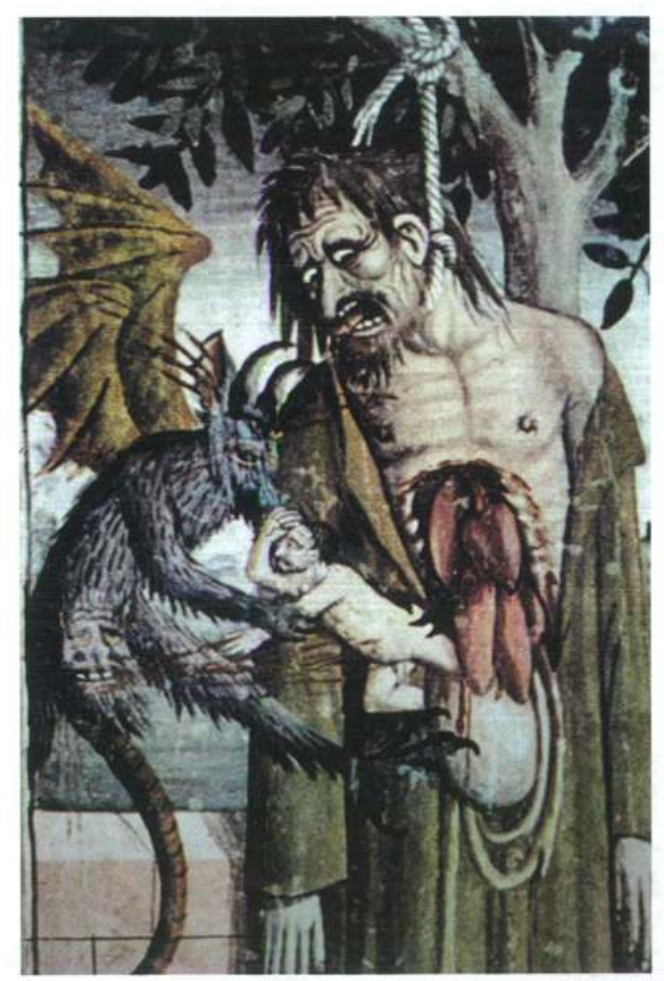

L'âme traîtresse de Judas sortant de son ventre ouvert (fresque peinte par G. Canavesio, Notre-Dame des Fontaines à La Brigue, Alpes-Maritimes, fin du XVe siècle).

Fig. 5

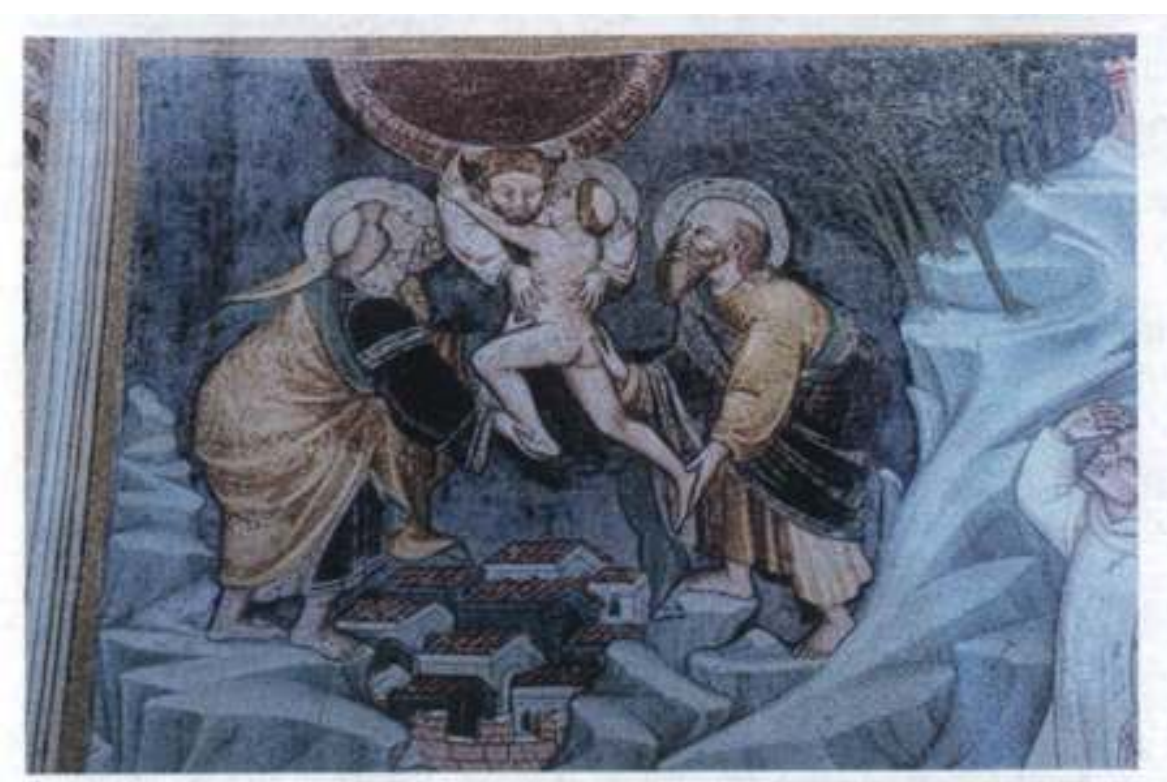

L'âme sainte de Thomas d'Aquin élevée jusqu'au ciel par saint Pierre et saint Paul (fresques de l'église Santa Maria in Piano à Loreto Aprutino, Abruzzes, vers 1420).

Pour synthétiser cette dernière partie, on reviendra sur la portée de la rédemption du corps glorieux et de l'assomption divine de l'homme. Cette élévation de la créature 
jusqu'à son Créateur et du corps de glaise jusqu'à la vertu de l'âme combine un double aspect contradictoire : elle élève le plus bas jusqu'au plus haut et paraît transcender les dualités hiérarchiques, mais à la condition expresse que le plus bas fasse montre d'obéissance et de soumission. La perspective de cette assomption peut paraître d'autant plus étonnante que le rapport Dieu/homme se formule au Moyen Âge comme un rapport entre Dominus et homo, soit les termes mêmes de la relation de domination entre le seigneur féodal et ses dépendants. Il convient donc de ne pas oublier que la jonction des extrêmes doit se réaliser dans l'utopie de l'autre monde, ce qui garantit le respect des prééminences terrestres, du moins tant que le millénarisme ne vient pas précipiter les temps. Hors d'une telle contestation, le rapport d'inversion que la doctrine établit entre ici-bas et au-delà enveloppe les hiérarchies sociales dans le manteau céleste de la communauté paradisiaque ${ }^{61}$ et inscrit la domination et le contrôle des corps terrestres dans l'attente d'un corps céleste glorifié. De même, l'assomption radicale des justes dans l'au-delà s'accompagne de l'exclusion d'une part considérable de l'humanité (nonbaptisés et mauvais chrétiens), rejetée dans les châtiments éternels de l'enfer. Telle est la dynamique double et complémentaire de la chrétienté et de l'institution ecclésiale qui la gouverne : à la fois excessivement aimante et intégratrice, c'est-à-dire aussi conquérante et évangélisatrice, et en même temps excluante et intolérante (de cette intolérance, dont M. Augé fait l'un des ressorts principaux de la constante victoire historique du christianisme sur les paganismes ${ }^{62}$ ).

Montrer que les représentations médiévales de la personne sont moins simples et moins dualistes qu'on ne le croit généralement ne vise nullement à les rapprocher des conceptions non-chrétiennes, en dépit de quelques contacts ponctuels. Si dans l'univers tzeltal analysé par Pedro Pitarch, «la représentation de la personne témoigne d'une relation réciproque avec le monde et d'un destin partagé avec d'autres êtres ${ }^{63}$ ", cette double interrelation avec le milieu et avec le groupe s'éclipse dans le christianisme au profit d'un lien privilégié entre l'âme et Dieu. À l'inverse, la conception chrétienne de la personne, unifiée et individualisée à travers son rapport à Dieu, est l'un des aspects qui s'impose le plus difficilement dans les mentalités indiennes ${ }^{64}$.

Cette relation entre la personne et le Dieu chrétien est généralement tenue pour une des voies par lesquelles avance le processus de l'individuation chrétienne, depuis les Confessions d'Augustin, qui se découvre comme sujet dans le sombre miroir que Dieu tend à son âme, jusqu'à la généralisation du souci intime de soi, que l'Église impose à partir du $\mathrm{XIII}^{\mathrm{e}}$ siècle notamment à travers l'obligation de la confession annuelle. Encore pourraiton suggérer que cette relation n'est pas sans ambiguïté, car en accentuant l'identité de toutes les âmes, unies par leur commune ressemblance en Dieu, elle peut aussi conforter l'affirmation de la communauté ecclésiale. En outre, il faut souligner que le lien entre l'âme et Dieu est largement médiatisé par les clercs, proclamés «médecins de l'âme » et spécialistes obligés de cette relation. Quoiqu'il en soit, ce n'est pas cet aspect que l'on a voulu analyser ici, préférant insister sur la pertinence sociale des conceptions de la personne (étant entendu que cette seule optique ne saurait rendre compte de tous ses aspects et variantes). On se rapproche ainsi de la démarche de P. Pitarch, lorsqu'il établit une homologie entre la personne et le village tzeltals - le cœur et la place étant castillans, le corps et les habitations indigènes (et on notera que son modèle de la personne tzeltale fonctionne de manière étonnamment proche de celui qui est proposé ici pour la personne chrétienne: fondamentalement duel en ceci que la conjonction d'une intériorité castillane et d'une extériorité indigène s'opère sans aucun mélange ni métissage, il est 
cependant préservé de tout dualisme par la figure du pli qui permet de penser l'articulation de deux contraires séparés $\left.{ }^{65}\right)$. De même, on peut voir une équivalence entre la personne chrétienne et la représentation fondamentale de l'Église, non seulement parce que la dualité corps/âme renvoie à la séparation clercs/laïcs, mais surtout parce que la dynamique qui conduit à la réalisation du corps glorieux des élus est celle qui anime toute l'organisation ecclésiale de la société.

À cet égard, il ne reste plus qu'à évoquer la logique d'un processus historique qui se fraie son chemin, à travers maints détours et contradictions. Schématiquement, on situera après les premiers siècles du christianisme où le dualisme le plus rude est porté par une logique de rupture avec la société romaine, un Haut Moyen Âge ascétique et monastique, valorisant particulièrement la fuite du monde et admettant des accents assez dualistes, comme chez Grégoire le Grand, non sans être poussé dans le même mouvement à penser l'homme à travers divers mélanges de l'esprit et de la matière. En revanche, la Réforme grégorienne s'efforce, sur tous les plans, à une distinction franche du spirituel et du matériel, qui à la fois les sépare et les articule hiérarchiquement, d'où un approfondissement du dépassement du dualisme, déjà engagé antérieurement mais qui s'affirme aux XII ${ }^{\mathrm{e}}$ XIII ${ }^{\mathrm{e}}$ siècles et trouve son apogée avec Thomas d'Aquin. Par la suite, l'unité thomiste s'affaiblit ; les disjonctions s'imposent peu à peu, et le dualisme trouvera finalement avec Descartes une forme radicale, qui a fortement pesé sur la conscience occidentale. Ainsi, c'est sans doute le Moyen Âge central, époque de l'Église triomphante, qui aura été la période la moins dualiste de l'histoire du christianisme, celle qui était la mieux à même d'éprouver l'unité psychosomatique de la personne que les conceptions modernes nous restituent d'une autre manière - et ce, parce que ce modèle était alors le plus pertinent pour penser le corps social et ecclésial, à la fois dans ses hiérarchies et dans son utopie communautaire ${ }^{66}$.

\section{NOTES}

1. Alfredo López Austin, Cuerpo humano e ideología. Las concepciones de los antiguos nahuas (1980), $3^{\mathrm{e}}$ éd., México, U.N.A.M. 1989 (citation, p. 197) ; Pedro PITARCH RAMÓN, Ch'ulel : una etnografía de las almas tzeltales, México, FCE, 1996.

2. Marc AUGÉ, Génie du paganisme, Paris, Gallimard, 1982, chap. 2 et 6 .

3. Marcel MAUSS, "Une catégorie de l'esprit humain : la notion de personne, celle de moi » (1938), repris dans Sociologie et anthropologie, Paris, P.U.F., 1985, PP. 331-362. Pour l'historiographie de l'émergence médiévale de l'individu, voir notamment Caroline W. BYNuM, "Did the Twelfth Century Discover the Individual? ", repris dans Jesus as mother. Studies in the Spirituality of the High Middle Ages, Berkeley, California University Press, 1982, pp. 82-109 ; JeanClaude sснмітт, «La découverte de l'individu, une fiction historiographique ? », in Pierre MENGAL et Françoise PAROT (éds.), La fabrique, la figure et la feinte. Fictions et statut de la fiction en psychologie, Paris, 1989, pp. 213-236 ; Aaron GOUREVITCH, Les origines de l'individualisme européen, Paris, Seuil, 1997.

4. Émile DURKHEIM, Les formes élémentaires de la vie religieuse, chap. 8, Paris, 1912. 
5. Peter BROWN, Le renoncement à la chair. Virginité, célibat et continence dans le christianisme primitif, trad. française, Paris, Gallimard, 1995, p. 50.

6. AUGUSTIN, déjà, souligne qu'il nomme mens ce que Paul appelle spiritus; De Genesi ad litteram. XII, 24, éd. Pierre AGAËsSE et André solignac, Bibl. Augustinienne, t. 49, Paris, Desclée, 1972, p. 417. Voir aussi M. D. CHENU, Saint Thomas d'Aquin et la théologie, Paris, Seuil, 1959, pp. 84-86.

7. Pour ces conceptions ternaires - et pour l'analyse d'ensemble -, voir les travaux de JeanClaude schmitt, "Le corps en chrétienté », in Maurice GODELIER et Michel PANOFF (éds.), La production du corps. Approches anthropologiques et historiques, Paris, Archives contemporaines, 1998, pp. 339-355, et "Corps et âme ", in Jacques LE GOFF et Jean-Claude SCHMITT (dirs.), Dictionnaire raisonné du Moyen Âge, Paris, Fayard, 1999. Voir aussi Michel Fromaget, Corps, âme, esprit. Introduction à l'anthropologie ternaire, Paris, Albin Michel, 1991.

8. Édouard-Henri WEBER, La personne humaine au XIII siècle, Paris, Vrin, 1991, p. 150. Dans son commentaire sur le De Trinitate de Boèce, il défend également que l'âme sensitive et l'âme intellective appartiennent à une seule et même essence, ibid., pp. 156-157.

9. Édouard-Henri WEBER, ibid., p. 125.

10. Voir Pedro PITARCH, Ch'ulel, op. cit., ainsi que l'article publié ici-même.

11. Serait-elle aussi plus directement influencée par elle ? Il semble plutôt que les missionnaires chrétiens se soient employés à réduire les composantes spirituelles de la personne à une seule âme (communication orale de Pedro Pitarch). En outre, cette dualité n'est pas mentionnée dans l'étude d'un autre municipe tzeltal (Pinola), dont les conceptions sont par ailleurs très proches de celles de Cancuc ; cf. Esther HERMITTE, Control social y poder sobrenatural en un pueblo contemporaneo (1970), 2 è éd., Tuxla Gutierrez, Instituto chiapaneco de cultura, 1992.

12. A. C. PEGIS, "The Separated Soul and its Nature in Saint Thomas", in S. Thomas Aquinas. 1274-1974. Commemorative Studies, Toronto, 1974, I, pp. 131-158.

13. Je renvoie à «Les représentations de l'au-delà: processus de localisation et formes communautaires ", communication à la table-ronde La spatialisation du sacré dans l'Occident latin, Auxerre, mars 1997.

14. Léon ELDERS, La philosophie de la nature chez saint Thomas d'Aquin, Paris, Éditions Pierre Téqui, 1994, p. 336.

15. Peter BROWN, op. cit., p. 282.

16. Jacques LE GOFF, « Anima », Enciclopedia dell'arte medievale, Rome, 1991, I, pp. 798-804 (voir par exemple chez Hugues de Saint-Victor, dont le De claustra animae fait du cœur la demeure de l'âme).

17. Guillaume de Diguleville, Pèlerinage de l'âme, J.J. STÜRZINGER éd., Londres, 1895, vers 6769-6912. Il suit ici le De spiritu et anima du XII ${ }^{\mathrm{e}}$ siècle.

18. Thomas d'Aquin, Summa Theologiae, Ia, qu. 10, art. 1, 4.

19. Pour l'affirmation du thème de l'ange gardien au XI ${ }^{\mathrm{e}}$ siècle, cf. Philippe FAURE, «L'homme accompagné. Origine et développement du thème de l'ange gardien en Occident ", Cahiers de Saint-Michel de Cuxa, 28, 1997, pp. 199-216; pour le diable personnel, je renvoie à "Diable », in Jacques LE GOFF et Jean-Claude schмiтt (dirs.), Dictionnaire raisonné de l'Occident médiéval, op. cit., pp. 260-272.

20. Ce paragraphe a été rédigé avant d'avoir connaissance de la communication de Pedro Pitarch, et notamment du texte de l'évêque chiapanèque Nunez de la Vega, mettant en scène son combat contre l'assimilation indigène entre ange gardien et nagual (être compagnon de la personne dont les labs de Cancuc sont une variante). Le rapprochement proposé ici y trouve une pertinence inespérée.

21. Pour un exposé plus complet sur cette question, je renvoie à «La parenté partagée: engendrement charnel et infusion de l'âme ", in Caria CASAGRANDE, Silvana VECCHIO, éds., Anima e 
corpo nella cultura medievale, Atti del V Convegno di studi della società italiana per lo studio del pensiero medievale (Venezia, 1995). Florence, SISMEL, edizione del Galluzzo, 1999, pp. 123-137.

22. Voir Guy DURAND, Anthropologie sexuelle et mariage chez saint Thomas d'Aquin, thèse dactyl., Faculté de théologie de Lyon, 1966-1967, pp. 94-97, et Summa Theologiae, Ia, qu. 118, art. 1-2.

23. Paris, Bibliothèque de l'Arsenal, ms. 5206, f. 174. Pour une analyse complète de cette miniature, voir « La parenté partagée ", art. cité. Le seul autre exemple connu, tiré du Scivias de Hildegarde de BINGEN, est analysé par Jean-Claude SCHMITT, «Le corps en chrétienté », art. cité, pp. 340-341.

24. On voit que le triomphe du créationisme est total, balayant l'objection qui constituait le fondement de l'hypothèse d'une création originelle, et selon laquelle toute création divine était achevée au terme des six premiers jours.

25. LE GOFF, La naissance du purgatoire, Paris. Gallimard, 1981. Pour les représentations de l'enfer, je renvoie à Les justices de l'au-delà. Les représentations de l'enfer en France et en Italie (XII ${ }^{e}-\mathrm{XV}^{e}$ siècles), BEFAR, 279, Rome, 1993.

26. Jean-Claude sснмітт, Les revenants. Les vivants et les morts dans la société médiévale, Paris, Gallimard, 1994. Sur la logique spatialisatrice de la société féodale, cf. Alain GUERREAU, « Quelques caractères spécifiques de l'espace féodal européen ", in Neithard BULST, Robert DESCIMON, Alain GUERREAU (éds.), L'État ou le Roi. Les fondations de la modernité monarchique en France (XIV ${ }^{e}$-XVIII siècles), Paris, EHESS, 1996, pp. 85-101.

27. Jean-Pierre VERNANT, «Aspects de la personne dans la religion grecque », repris dans Mythe et pensée chez les Grecs, rééd., Paris, 1980, II, p. 89.

28. Pedro PITARCh insiste sur le lien entre la décomposition de la personne lors de la mort et l'absence de préoccupation pour l'au-delà chez les tzeltals. Mario H. RUZ repère néanmoins, chez les mayas yucatèques, une plus grande continuité par-delà la mort, qui caractérise également les conceptions nahuas analysées par Alfredo LóPEZ AUSTIN, Tamoanchán y Tlalocán, México, FCE, 1994.

29. Guibert de NOGENT, De vita sua, I, 18, E. R. LABANDE éd., Paris, Les Belles-lettres, 1981, p. 150.

30. Retractationes, I, 3, Bibl. Augustinienne, p. 278 ; cf. Édouard-Henri WEBER, op. cit., pp. 27-28.

31. Hildegarde de BINGEN, Les causes et les remèdes, trad. P. Monat, Paris, Millon, 1997, pp. 81-82.

32. Ibid., p. 15.

33. Selon l'expression très dualiste du De spiritu et anima, ch. 14, PL, 40, c. 790, cité par ÉdouardHenri WEBER, op. cit., p. 53.

34. Pierre lOMBARD, Liber Sententiarum, II, 1, 6, 4, cité par Édouard-Henri WEBER, op. cit., p. 60.

35. Caroline W. BYnum, The Resurrection of the Body in Western Christianity, 200-1336, New York, Columbia University Press, 1995, p. 331.

36. Édouard-Henri WEBER, op. cit., p. 102.

37. Marie Dominique CHENU, Saint Thomas d'Aquin, op. cit., p. 122.

38. «Pour la plénitude de son activité intellective, elle a besoin d'exercer des opérations sensitives au moyen du corps ", cité par Édouard-Henri WEBER, op. cit., p. 152.

39. On pense au livre de Édouard-Henri WEBER, largement utilisé ici pour ses qualités d'analyse, mais qui me paraît fausser quelque peu la perspective, dès lors que pour lui la ligne de démarcation entre le dualisme et son dépassement sépare Thomas de tous les autres théologiens. 40. Caroline W. Bynum, The Resurrection, op. cit.

41. Ainsi, les tzeltals de Cancuc, pourtant peu enclins à se moquer des choses religieuses, trouvent dans l'idée chrétienne de la résurrection des corps une occasion de rire à gorge déployée ; Pedro PITARCH, Ch'ulel, op. cit., p. 210.

42. Augustin, De civitate Dei, 22, 19, éd. B. Dombart et A. Kalb, Bibl. Augustinienne, 37, Paris, Desclée, 1960.

43. Calixta Guiteras holmes, Los peligros del alma. Visión del mundo de un tzotzil, México, F.C.E., 1965, p. 125 ; ainsi que André AUBRY, Les tzotzils par eux-mêmes, Paris, L'Harmattan, 1988, p. 59. On 
reconnaîtra volontiers que le rapprochement proposé est bien incertain - d'autant plus que Alfredo LóPEZ AUSTIN (Tamoanchán y Tlalocán, op. cit., p. 211) repère dans les croyances nahuas une valorisation spécifique des ongles et des cheveux, perçus comme les symboles sensibles de la croissance du corps humain. La coutume évoquée ici pourrait donc trouver son sens dans la logique des conceptions mésoaméricaines.

44. «Caro spiritalis, sed tamen caro, non spiritus » dit AUGUSTIN, pour qualifier le corps spirituel des élus (De civitate Dei, 22 21, éd. citée). Pour Thomas d'Aquin, « il est hérétique de prétendre qu'à la résurrection le corps deviendra esprit selon le mot de saint Paul, entendu littéralement ", Summa Theologiae, III, Supp., qu. 83.

45. À la fin du XI ${ }^{\mathrm{e}}$ siècle, Anselme de Canterbury établit le schéma, promis à un grand succès, des sept béatitudes de l'âme et des sept béatitudes du corps (pulchritudo, velocitas, fortitudo, libertas, impassibilitas, voluptas, aelernitas vitae), cf. De beatitudine coelestis patriae, chap. I-XIV, PL, 159, c. 596-598, ainsi que Proslogion, Michel CORBIN, éd., chap. 25-26, Paris, Cerf, 1986, pp. 118-122. Les scolastiques $\mathrm{du}$ XIII ${ }^{\mathrm{e}}$ siècle préfèrent toutefois condenser l'analyse en quatre béatitudes (claritas, agilitas, subtilitas, impassibilitas), par exemple chez Thomas d'Aquin, Summa Theologiae, III, Supp., qu. 82-85.

46. Mario H. Ruz, Copanaguastla en un espejo. Un pueblo tzeltal en el virreinato, San Cristóbal de las Casas, UNACH, 1985, pp. 215-217.

47. Peter BROWN, Le renoncement, op. cit., p. 76.

48. Des causes et des remèdes, op. cit., p. 85.

49. Christian trottmann, La Vision béatifique, des disputes scolastiques à sa définition par Benoît XII, BEFAR, Rome, 1995.

50. Je dois ce rapprochement entre la conception chrétienne du corps glorieux des élus et la notion grecque du sur-corps des dieux, analysée par J.-P. Vernant, à un commentaire de Françoise Frontisi (Londres, 1997).

51. Je renvoie à mon compte-rendu in Annales, H.S.S., 1996, 1, pp. 135-139.

52. Bonaventure, Breviloquium, pars VII, ch. VII, éd. L. Prunière, Paris, 1967, pp. 315-319.

53. Voir Anita GUERREAU-JALABERT, «Spiritus et caritas. Le baptême dans la société médiévale », in Françoise HÉRITIER-AUGÉ, Elisabeth COPET-ROUGIER (éds.), La parenté spirituelle, Paris, Archives contemporaines, 1996, pp. 133-203.

54. Cf. Jacques GAUDEMET, Le mariage en Occident. Les mœurs et le droit, Paris, 1987.

55. Voir Jean-Claude schmiтt, Les revenants, op. cit.

56. Michel vovelle, La mort et l'Occident de 1300 à nos jours, Paris, Gallimard, 1983, pp. 40-56 (pour d'autres exemples).

57. Emmanuel LE ROY LADURIE, Montaillou, village occitan de 1294 à 1324, $2^{\mathrm{e}}$ éd. Paris, Gallimard, 1982, p. 596, ainsi que pp. 589-595 pour la corporalité des âmes (« elles ont une chair, des os et tous les membres ", dit G. FORT).

58. Guibert de NOGENT, De pignoribus sanctorum, IV, 4, PL, 156, c. 675-678, cité par Jean-Claude sCHміт, Les revenants, p. 225.

59. Pour une analyse complète de cette image, cf. Jerôme BASCHET et J.-C. BONNE, « La chair de l'esprit (à propos d'une image insolite de Thomas d'Aquin) », in Jacques REVEL et Jean-Claude schmiтt, éds ; L'ogre historien. Autour de Jacques Le Goff, Paris, Gallimard, 1998, pp. 193-221.

60. La notion de condescensio - l'attention que les êtres spirituels prêtent au monde charnel - est analysée dans un tout autre contexte par Peter BROWN, L'essor du christianisme occidental, Paris, Seuil, 1997, p. 173.

61. Sur la communauté fraternelle du paradis et ses formulations textiles, je renvoie à Jérôme BASCHET, Le sein du père. Abraham et la paternité dans l'Occident médiéval, Paris, Gallimard, 2000.

62. Marc AUGÉ, Génie du paganisme, op. cit., pp. 78-79.

63. Pedro PITARCH, Ch'ulel, op. cit., p. 107. 
64. Serge GRUZINSKI, La colonisation de l'imaginaire, Paris, Gallimard, 1988, p. 229; Juan Pedro VIQUEIRA, «Qu'y avait-il derrière le petate de la chapelle de Cancuc ?», repris dans Une rébellion indienne au Chiapas : 1712, Paris, L'Harmattan, 1999.

65. Pedro PITARCH, Ch'ulel, op. cit., pp. 185-187, et pour la figure du pli, pp. 251-256.

66. L'actualité mexicaine suggère toutefois d'étonnantes continuités. Ainsi, pendant la rédaction du présent texte, on a pu lire un point de vue publié en défense de Mgr. Samuel Ruiz, quelques jours après l'autodissolution de la "Comisión Nacional de Intermediación", dont il était président (Miguel CONCHA, La Jornada, 13.06.98). L'article s'ouvre par une définition chrétienne de «la personne humaine, considérée comme un tout, et non comme une âme qui habite un corps » - laquelle permet ensuite de justifier l'engagement politique de l'évêque de San Cristóbal de las Casas, loué pour sa fidélité à la tradition des grands prélats des $\mathrm{IV}^{\mathrm{e}}-\mathrm{V}^{\mathrm{e}}$ siècles, totalement impliqués dans les affaires du monde. Ainsi, ce modèle de la personne est clairement associé à une conception de l'Eglise, inaugurée par Augustin, réalisée durant l'époque médiévale et qui aujourd'hui encore semble ne pas avoir cessé d'être vivante.

\section{RÉSUMÉS}

Le présent article vise à montrer que les conceptions de la personne humaine dans l'Occident médiéval sont plus complexes que ne le laisse penser leur apparence de simple dualité. Outre différents aspects qui peuvent s'ajouter à la dualité âme/corps, il convient de prêter une grande attention au type de rapport établi entre l'âme et le corps. On constate alors que la dualité est pour le christianisme médiéval un moyen de se distinguer radicalement du dualisme, en permettant de penser l'articulation et l'union nécessaire de l'âme et du corps. Au fil des siècles qui mènent jusqu'au Moyen Âge central, cet aspect ne fait que se renforcer; il conduit à proclamer avec emphase le caractère positif de l'union de l'âme et du corps, seule capable de constituer véritablement la personne humaine, qui doit être reformée à la fin des Temps grâce à la résurrection des corps. Enfin, on argumentera que le corps glorieux des élus, et plus largement l'articulation du corporel et du spirituel promue par le discours théologique, constituent un modèle idéal de la société chrétienne. Toute la logique de la chrétienté médiévale, entièrement structurée par l'Institution ecclésiale, dépend de sa capacité à articuler positivement le spirituel et le corporel, c'est-à-dire à spiritualiser le corporel. Quelles que soient les ambiguïtés et les contestations possibles, l'image de la personne et l'unité psychosomatique pensées par la scolastique se révèlent être un instrument puissant pour fonder le corps social et ecclésial, à la fois dans ses hiérarchies et dans son utopie communautaire.

The author tries to show that the conception of the human person in the West in medieval times is more complex than its apparent simple duality would have us believe. In addition to the various aspects that can be added to the duality soul/body, close attention should be given to the type of relationship established between the soul and the body. One thus realizes that for medieval christianity this duality offered a means to differentiate itself radically from dualism, and to establish the articulation and necessary union between soul and body. This aspect was constantly strengthened along the centuries leading to the middle medieval period, and brought a strong emphasis on the positive dimension of the union of body and soul, which alone really constitutes the human person, and which should be recreated at the end of times through bodily resurrection. Finally, the author argues that the glorious body of the elect, and more generally, 
the articulation of the corporal and spiritual dimensions presented in the theological discourse, represent an ideal model of Christian society. The whole logic of medieval Christendom, entirely structured by the Church, depended on its ability to articulate positively the spiritual and corporal dimensions, i.e. to spiritualize the body. Whatever their ambiguities and the possible counter-arguments, the image of the person and the psychosomatic unity elaborated by scholasticism appear as a powerful tool that gives a foundation to the social and ecclesiastical body, to its hierarchy as well as its communitarian utopianism.

El presente artículo busca demostrar que las concepciones de la persona humana en el Occidente medieval son mucho más complejas de lo que deja pensar su aparente dualidad. Además de varios aspectos que pueden añardirse a la dualidad alma/cuerpo, es indispensable prestar atención al tipo de relación establecido entre el alma y el cuerpo. Esto permite constatar que la dualidad sirve al cristianismo para diferenciarse radicalmente del dualismo, afirmando la articulación y la necesaria unión entre el alma y el cuerpo. Este aspecto se hace cada vez más fuerte, conforme avanzamos hacia la Edad Media central, y lleva a proclamar con insistencia el carácter positivo de la union entre el alma y el cuerpo, indispensable para definir la persona humana y que debe ser reformada al Fin de los Tiempos, gracias a la resurección de los cuerpos. Por último, se argumenta que el cuerpo glorioso de los elegidos, y más generalmente la articulación de lo corporal y lo espiritual promovida por et discurso teológico, constituyen un modelo ideal de la sociedad cristiana. Toda la lógica de la cristiandad medieval, estructurada enteramente por la institución eclesial, depende de su capacidad a articular positi-vamente lo espiritual y lo corporal, es decir a espritualizar lo corporal. Cuales que sean las ambigüedades y las contestaciones posibles, la imagen de la persona y la unidad psicosomática pensadas por la escolástica parecen ser una herramienta eficaz para fundar el cuerpo social y eclesial, tanto en sus jerarquias como en su utopía comunitaria.

\section{AUTEUR}

\section{JÉRÔME BASCHET}

Centre de Recherches Historiques, E.H.E.S.S. 